Check for updates

Cite this: Chem. Commun., 2021,

57, 1540

\section{Introduction to (photo)electrocatalysis for renewable energy}

\author{
Abhishek Dey, (D) *a Frances A. Houle, (D) $\star^{b}$ Carolyn E. Lubner, (D) *c \\ Marta Sevilla (iD *d and Wendy J. Shaw (D)*e
}

DOI: $10.1039 / \mathrm{d} 0 \mathrm{cc} 90530 \mathrm{e}$

rsc.li/chemcomm

Humanity is facing a significant and growing energy demand, necessitating a new energy paradigm built on clean and abundant renewable energy. ${ }^{1}$ Within this context, electrocatalysis and photoelectrocatalysis hold great promise for enabling renewable energies such as solar and wind power to overcome the transient nature of their energy output. ${ }^{2-6}$ Developing catalysts that operate efficiently and selectively, and that are also robust, has been the focus of much research. This themed issue brings together the latest advances in the fundamental and applied science addressing the synthesis, characterization, mechanisms, and performance of (photo)electrocatalysts for energy storage and conversion of small molecules into useful specialty and commodity chemicals and fuels. The electrocatalysts and photoelectrocatalysts highlighted in this issue include metalloenzymes, homogeneous and supported molecular catalysts, and

\footnotetext{
${ }^{a}$ Indian Association for the Cultivation of Science, Kolkata, WB, 700032, India.

E-mail:abbeyde@gmail.com

${ }^{b}$ Liquid Sunlight Alliance and Chemical Sciences Division, Lawrence Berkeley National Laboratory, Berkeley, CA 94520, USA. E-mail: fahoule@lbl.gov ${ }^{c}$ Biosciences Center, National Renewable Energy Laboratory, Golden, CO 80401, USA.

E-mail: Cara.Lubner@nrel.gov

${ }^{d}$ Instituto de Ciencia y Tecnología del Carbono, INCAR-CSIC, Oviedo 33011, Spain.

E-mail: martasev@incar.csic.es

${ }^{e}$ Pacific Northwest National Laboratory, Richland, WA, 99354, USA. E-mail: Wendy.Shaw@pnnl.gov
}

diverse heterogeneous nanoparticulate and thin film systems. The reports span across basic and applied studies and encompass both experimental and theoretical investigations.

\section{Metalloenzymes}

In this issue, advances in understanding the mechanistic principles of electrocatalytic and photocatalytic enzymes are presented. Enzymes serve as a model for molecular and synthetic catalysts for storing and interconverting energy. They are highly selective and efficient, providing design principles for developing catalysts that can be used in an industrial capacity. Hydrogenase, CO dehydrogenase, formate dehydrogenase, nitrogenase, and others demonstrate that non-precious metals and mild conditions (room temperature and atmospheric pressure) are capable of achieving high catalytic performance as discussed in the article by Minteer and co-workers (DOI: 10.1039/ D0CC02672G). One desirable feature of enzymes is that they often function at the operating potential of the reaction, or at minimal overpotentials. Furthermore, enzymes can coordinate multi-electron catalysis in the absence of unwanted side reactions through a variety of mechanisms; one such example is detailed in the report by Shima and co-workers (DOI: 10.1039/ D0CC04557H). Understanding what features drive the reaction one way versus the other, i.e. catalytic bias, also offers important insight into controlling the reaction progression, as discussed by Peters (DOI: 10.1039/D0CC07062A). While enzymes display the aforementioned favorable characteristics, they commonly suffer from inactivation under practical conditions and thus reinstating their reactivity often requires ingenious designs such as incorporation into a redox-active hydrogel as reported by Plumeré and co-workers (DOI: 10.1039/D0CC03155K). The more we understand the underpinnings of these processes, the better we are able to implement them into other types of catalysts, with the goal to ultimately achieve faster, more efficient, and more controllable synthetic catalysts.

\section{Bioinspired molecular catalysts and MOF catalysts}

One of the goals of synthesizing new molecular and bioinspired (photo)electrocatalysts is to try to achieve high rates at low overpotentials. This can be based on best principles from the molecular catalyst community or by implementing either functional or structural features from enzymes. The work by Apfel and co-workers (DOI: 10.1039/D0CC05229A) and Hardré and co-workers (DOI: 10.1039/ D0CC04174B) provides examples of such efforts. A primary advantage of molecular 
catalysts is that the ligand structure surrounding the active center can be tuned to control reactant supply as well as selectivity when multiple reaction pathways are available. This is nicely demonstrated in the paper by Brudvig and co-workers (DOI: 10.1039/D0CC03207G) where selectivity in $\mathrm{CO}_{2}$ reduction is reported. In general, these catalysts are slower and less energy efficient compared to enzymes, although some systems are beginning to approach enzymatic performance. ${ }^{7}$ A feature that is often mimicked is proton relays, whether fixed, flexible, or even part of the solvent as demonstrated in the paper by Dutta and co-workers (DOI: 10.1039/D0CC05786J). A new avenue allowing precise control over the catalytic active site is the use of metal-organic frameworks (MOFs). The controlled assembly of catalytic centers allows tunability of substrate access channels as detailed in the article by Das and co-workers (DOI: 10.1039/D0CC03659E). They describe integration strategies that will allow their potential as electrocatalysts for water oxidation to be realized.

\section{Heterogeneous catalysts and sensitizers}

Unlike molecular catalysts, which can be designed for precise control over a specific chemical reaction pathway, heterogeneous catalysts typically comprise a broad diversity of reactive sites placed close together and can involve multiple elements in crystalline or amorphous form. These features can facilitate multistep reactions, such as $\mathrm{O}_{2}$ reduction, water oxidation and $\mathrm{CO}_{2}$ reduction, but may introduce challenges for selective formation of a desired product. Progress toward efficient and selective reduction and subsequent valorization of the reduced forms of $\mathrm{CO}_{2}$ using heterogeneous catalysts will open an attractive avenue for carbon fixation as demonstrated by Figueiredo and co-workers (DOI: 10.1039/D0CC04231E). Reisner and co-workers (DOI: 10.1039/D0CC01686A) describe the reverse process, where photocatalysis is used for reforming biomass to generate $\mathrm{H}_{2}$ and sugars. Electrocatalytic and plasmonic nanoparticles and $0 \mathrm{D} / 2 \mathrm{D}$ hybrid materials are being explored to improve activity and selectivity. The papers by Pescarmona (DOI: 10.1039/ D0CC06818G), McCloskey (DOI: 10.1039/ D0CC03215H), Matson (DOI: 10.1039/ D0CC03163A), and Shaijumon (DOI: 10. 1039/D0CC03053H) and their co-workers present attractive developments in that area. Molecular catalysts can be immobilized to result in stable heterogeneous catalytic systems and a convenient method to covalently attach catalysts to ITO surfaces is demonstrated by Brudvig and co-workers (DOI: 10.1039/D0CC03631E). Mulfort and coworkers (DOI: 10.1039/ D0CC05972B) demonstrated photoinduced charge transfer that can drive reduction reactions from sensitizers immobilized in nanoporous metal oxide environments.

Carbon materials have proved their efficiency as electrocatalyst supports, improving both the catalytic activity and stability of the active phase through different mechanisms (e.g., decreased size, charge transfer or surface interactions). In particular, dispersion of the active phase is key to enhancing activity, the extreme case being single atom catalysts. Indeed, single atom catalysts on carbon materials have attracted much attention in recent years owing to their superb activity at very low loadings of active sites. Such examples are illustrated in the articles by Oh and co-workers (DOI: 10.1039/D0CC04752J) and Elbaz and co-workers (DOI: 10.1039/D0CC03122D). One challenge to be solved is the synthesis of high content single atom supported catalysts for practical applications. The intrinsic activity of doped carbon materials in several electrochemical reactions (ORR, OER, HER) has made them a very attractive metal-free option for sustainable energy production at an acceptable cost. Further understanding is necessary, particularly regarding control over the performance of active sites (dopants, chemical configuration, defects), which will rely on advancements in the synthesis of carbon materials with very precise control of their chemical and structural features, as well as on the design of new in-situ characterization techniques.

\section{Applications}

Ultimately, incorporating high-performing catalysts in electrolyzers, fuel cell systems, metal-air batteries and photoelectrochemical cells will help to enable renewable energy technologies. In each of these applications, several factors have limited their practical implementation, including the cost and scarcity of precious metal catalysts, the stability of the catalysts both during operation and in contact with electrolytes, and the high overpotentials required to generate the products of interest at practical rates. Each technology operates in a different current-voltage regime, placing unique demands on the chosen catalysts. For example, water splitting to form $\mathrm{H}_{2}$ and $\mathrm{O}_{2}$ can be performed with grid-attached electrolyzers, electrolyzers equipped with a separate photovoltaic system, and a system driven directly by absorption of solar radiation. Such applications have been discussed by Gómez and co-workers (DOI: 10.1039/D0CC04387G) for oxide electrode materials. The stability of mixed metal oxides for water oxidation as a function of acidic electrolyte composition has been discussed in the article by Nocera and co-workers (DOI: 10.1039/ D0CC03863F). The electrolyzer systems operate at high current density (0.5$2 \mathrm{~A} \mathrm{~cm}^{-2}$ ), while the solar driven system is in the range of $10-20 \mathrm{~mA} \mathrm{~cm}{ }^{-2}$. The variety of operating conditions place very different demands on catalyst activity. For systems operating at high current densities, control of resistances and catalyst overpotentials has also been a more recent focus, with important developments demonstrating the possibility of achieving energy efficient fuel cells. While the development of more efficient catalysts has been critical, advances in non-precious metal catalysts and in understanding how to control heterogeneous catalyst selectivity and stability and promote favorable charge transfer kinetics have also been crucial. The paper by Bhattacharyya and co-workers (DOI: 10.1039/D0CC03154B) illustrates this issue in $\mathrm{Zn}$ air batteries. Photocatalysts are at a much earlier stage of development for applications, and research into factors influencing their stability will 
be very valuable. Theoretical calculations can help predict the properties of energy harvesting systems as demonstrated by Ghosh (DOI: 10.1039/D0CC04702C) and Coridan (DOI: 10.1039/DOCC04229C) and their co-workers. Mechanistic understanding of catalysts is key to the development of selective and efficient catalysts which will require development of new experimental tools specifically for probing different aspects of electrocatalysis. This includes operando investigations as demonstrated by Yano and co-workers (DOI: 10.1039/ D0CC00692K), who use operando XAS, as well as post operation analysis as demonstrated by McCrory and co-workers (DOI: 10.1039/D0CC06513G). These investigations not only help characterize the material but also provide insight into the chemical processes that are rate limiting under operational conditions.

In summary, the advancements highlighted in this themed issue demonstrate how far we have come as a scientific community in understanding, designing, and building (photo)electrocatalysts. They leave no doubt that there is significant work left to do, but provide hope that with dedicated focus, highly efficient, high performing, and robust catalytic systems are a reality that we can achieve.

\section{Acknowledgements}

$\mathrm{AD}$ acknowledges the Department of Science and Technology, India. FAH acknowledges support by the Liquid Sunlight Alliance, which is supported by the U.S. Department of Energy (DOE), Office of Science, Office of Basic Energy Sciences (BES), Fuels from Sunlight Hub under Award Number DESC0021266. This work was authored in part by Alliance for Sustainable Energy, LLC, the manager and operator of the National Renewable Energy Laboratory for the U.S. Department of Energy (DOE) under Contract No. DE-AC36-08GO28308. Funding was provided to CEL by the U.S. DOE, Office of Science, Early Career Program. MS acknowledges support by the Spanish MCIU and regional FICYT. WJS acknowledges support by the US DOE, Office of Science, BES, Chemical Sciences, Geosciences, and Biosciences. The views expressed in the article do not necessarily represent the views of the DOE or the U.S. Government. The U.S. Government retains and the publisher, by accepting the article for publication, acknowledges that the U.S. Government retains a nonexclusive, paidup, irrevocable, worldwide license to publish or reproduce the published form of this work, or allow others to do so, for U.S. Government purposes.

\section{References}

1 https://www.energy.gov/science-innovation/ clean-energy.

2 Electrocatalysis for fuels, https://www.nat ure.com/collections/vnmbcthpkl.

3 M. T. Spitler, M. A. Modestino, R. G. Deutsch, C. X. Xiang, J. R. Durrant, D. V. Esposito, S. Haussener, S. Maldonado, I. D. Sharp, B. A. Parkinson, D. S. Ginley, F. A. Houle, T. Hannappel, N. R. Neale, D. G. Nocera and P. C. McIntyre, Practical challenges in the development of photoelectrochemical solar fuels production, Sustainable Energy Fuels, 2020, 4, 985-995.

4 Basic Energy Sciences Roundtable Liquid Solar Fuels, 2019.

5 S. Ardo, D. F. Rivas, M. A. Modestino, V. S. Greiving, F. F. Abdi, E. A. Llado, V. Artero, K. Ayers, C. Battaglia, J.-P. Becker, D. Bederak, A. Berger, F. Buda, E. Chinello, B. Dam, V. D. Palma, T. Edyinsson, K. Fujii, H. Gardeniers, H. Geerlings, S. M. H. Hashemi, S. Haussener, F. Houle, J. Huskens, B. D. James, K. Knorad, A. Kudo, F. P. Kunturu, D. Lohse, B. Mei, E. L. Miller, G. F. Moore, J. Muller, K. L. Orchard, T. E. Rosser, F. H. Saadi, J.-W. Schuttauf, B. Seger, S. W. Sheehan, W. A. Smith, J. Spurgeon, M. H. Tang, R. v. d. Krol, P. C. K. Vesborg and P. Westerik, Pathways to electrochemical solar-hydrogen technologies, Energy Environ. Sci., 2018, 11, 2768-2783.

6 DOE Explains...Solar Fuels, https://www. energy.gov/science/doe-explainssolar-fuels.

7 R. M. Bullock, J. G. Chen, l. Gagliardi, P. J. Chirik, O. K. Farha, C. H. Hendon, C. W. Jones, J. A. Keith, J. Klosin, S. D. Minteer, R. H. Morris, A. T. Radosevich, T. B. Rauchfuss, N. A. Strotman, A. Vojvodic, T. R. Ward, J. Y. Yang and Y. Surendranath, Using nature's blueprint to expand catalysis with earth-abundant metals, Science, 2020, 369, eabc3183. 